\title{
Solomon Echoes Extended to Soft Pulse Excitation*
}

\author{
Pascal P. Man \\ Laboratoire de Chimie des Surfaces, CNRS URA 1428, Université Pierre et Marie Curie, \\ 75252 Paris Cedex 05, France
}

Z. Naturforsch. 49a, 89-96 (1994); received July 23, 1993

Solomon echoes, produced by two in-phase pulses separated by a delay $\tau_{2}$, are derived in taking into account the first-order quadrupolar interaction during the pulses. Consequently, the results are valid for any ratio of the quadrupolar coupling, $\omega_{\mathrm{Q}}$, to the amplitude of the radiofrequency pulse, $\omega_{\mathrm{RF}}$. Six echoes are predicted after the second pulse: three, detected as inner satellite signals, occur at $\tau_{4}=\tau_{2}, \tau_{4}=2 \tau_{2}$ and $\tau_{4}=3 \tau_{2}$; the other three, detected as outer satellite signals, occur at $\tau_{4}=\tau_{2} / 2$, $\tau_{4}=\tau_{2}$, and $\tau_{4}=3 \tau_{2} / 2$. These echoes originate from the refocusing of single- and multi-quantum offresonance coherences developed during the first pulse. Four of them are allowed echoes, the other two are forbidden ones. However, there is no echo for the central transition. From a practical point of view this means that $\tau_{2}$ must be short compared to $T_{\mathrm{FID}}$, the duration of the FID of the central transition. The behavior of the echo amplitude versus the second pulse length is discussed for several ratios of $\omega_{\mathrm{Q}} / \omega_{\mathrm{RF}}$

Key words: Quadrupole effect, Spin-5/2, Spin echo NMR, Solomon echoes

\section{Introduction}

The first attempt to extract structural information in a spin- $5 / 2$ system from echoes, namely the distribution of electric-field-gradients (EFG), has been undertaken by Solomon [1, 2], followed by Dowley [3], Butterworth [4], Weisman and Bennett [5], and Sanctuary and coworkers $[6,7]$. The sequence consists of two radiofrequency pulses separated by a delay. As the first-order quadrupolar interaction $\left(H_{\mathrm{Q}}^{(1)}\right)$ has not been considered during the two pulses, four echoes out of six have been predicted: the four allowed echoes but not the two forbidden echoes. The two forbidden echoes, defined by Solomon [1, 2], correspond to the refocusing of multiquantum coherences (terms not yet used at that time) generated by the first pulse. Due to the fact that these coherences have not been considered in his computation, the associated echoes have not been predicted. However, all the six echoes have been observed: Allowed echoes are bell-shaped curves; forbidden echoes, also called sine echoes [6], are derivatives of bell-shaped curves [1,2]. This leads to the conclusion that bell-shaped echoes arise from hard

\footnotetext{
* Presented partially at the XIIth International Symposium on Nuclear Quadrupole Resonance Spectroscopy, Zürich, Switzerland, July 19-23, 1993.
}

Reprint requests to Dr. P. P. Man, Laboratoire de Chimie des Surfaces, CNRS URA 1428, Université Pierre et Marie Curie, 4 Place Jussieu, Tour 55, 75252 Paris Cedex 05, France.-E-mail: pm@ccr.jussieu.fr. pulse while additional derivative shaped echoes are observed when the pulses are soft [6]. Disregarding the first-order quadrupolar interaction during the pulses implies that the results are only valid for the "hard" pulse excitation (the quadrupolar coupling $\omega_{\mathrm{Q}}$ is supposed to be much smaller than the amplitude $\omega_{\mathrm{RF}}$ of the pulse), during which the evolution of the spin system is described simply by the Wigner rotation matrix. Recently, the spin-echo sequence used in different fields of magnetic resonance has been reviewed by leading experts [8].

The present paper extends the excitation condition to the "soft" pulse case by taking into consideration $H_{\mathrm{Q}}^{(1)}$ throughout the experiment. Furthermore, the computation starts from the thermal equilibrium of the spin system. This state is described by a density matrix $\varrho(0)=I_{z}$. In contrast, in the early papers $[1,2,4,5]$, the initial condition is described by $\varrho(0)=I_{x}$, corresponding to a state of the spin system after the first $\pi / 2$ pulse excitation. As a result, all the six echoes are revealed from our computation. In addition, each echo can take any shape ranging from a bell-shaped curve to a sine-shaped one, depending on the excitation conditions.

\section{Theory}

The energy levels of a spin- $5 / 2$ system are represented in Fig. 1, where the eigenstates of $I_{z},|m\rangle$ are redefined as: $|k\rangle=|I-m+1\rangle$, so $k=1, \ldots, 2 I+1$. On 


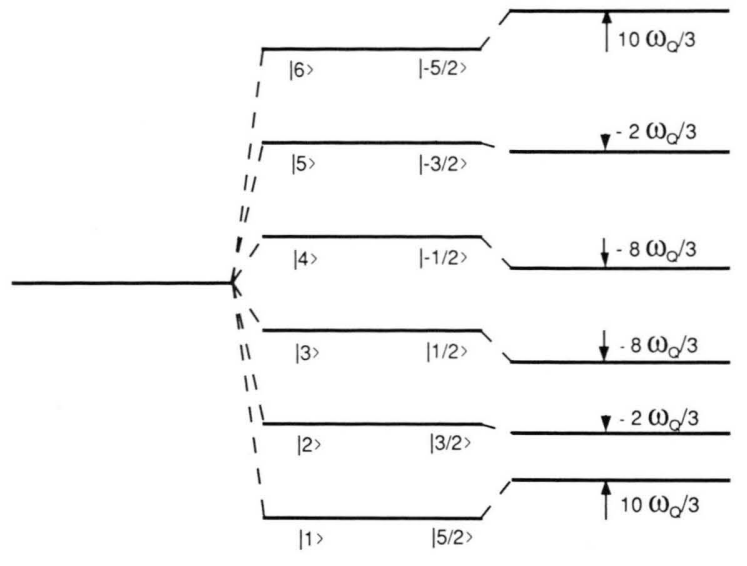

$$
\begin{array}{lll}
\mathrm{H}_{\mathrm{z}}=0 & \mathrm{H}_{\mathrm{z}} \neq 0 \quad \mathrm{H}_{\mathrm{z}}+\mathrm{H}_{\mathrm{Q}}^{(1)}
\end{array}
$$

Fig. 1. The six energy levels, their shifts and the two forms of eigenstates for spin-5/2 ( $H_{\mathrm{z}}$ means Zeeman interaction).

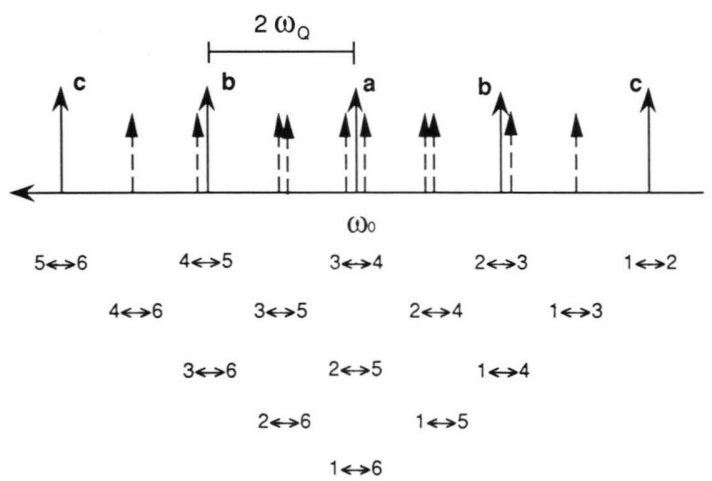

Fig. 2. The line positions associated with the transition $(i \leftrightarrow j)$ are schematically represented by arrows. The solid arrows, (a), (b) and (c), are $1 \mathrm{Q}$ coherences detected by a one-pulse experiment. The dashed arrows are MQ coherences not detectable by a one-pulse experiment. Their heights are meaningless.

the other hand, Fig. 2 shows the location of the lines in the spectrum: $(3 \leftrightarrow 4)$ the central transition, $(2 \leftrightarrow 5)$ the three-quantum transition ( $3 \mathrm{QT})$, and $(1 \leftrightarrow 6)$ the 5QT are unshifted by $H_{\mathrm{Q}}^{(1)} ;(2 \leftrightarrow 3)$ and $(4 \leftrightarrow 5)$ the inner satellite (I-S) transitions are shifted from the Larmor frequency $\omega_{0}$ by $\pm 2 \omega_{\mathrm{Q}} ;(1 \leftrightarrow 2)$ and $(5 \leftrightarrow 6)$ the outer satellite $(\mathrm{O}-\mathrm{S})$ transitions are shifted by $\pm 4 \omega_{\mathrm{Q}}$. Of course, only single-quantum (1 Q) transitions, represented by solid arrows, are observable directly with one-pulse excitation.

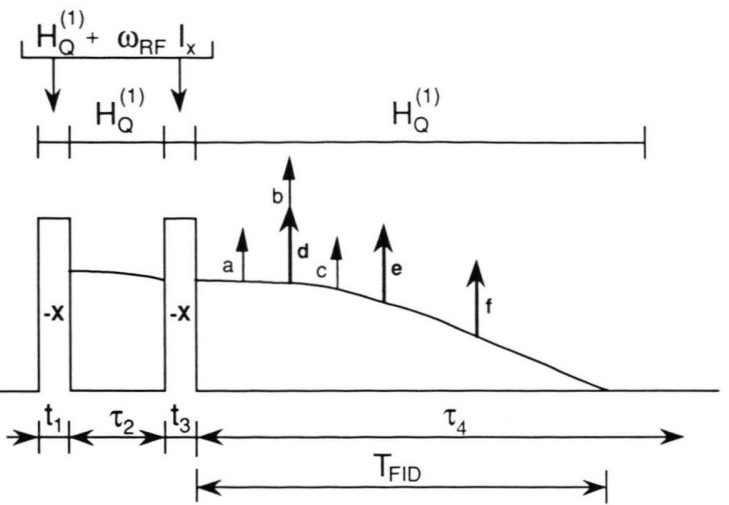

Fig. 3. Interactions considered and durations associated with a two $-x$ pulse sequence. Echoes are represented schematically by upward arrows whose heigh is meaningless. Echoes of the O-S transitions: (a) $\tau_{4}=\tau_{2} / 2$, (b) $\tau_{4}=\tau_{2}$, (c) $\tau_{4}=3 \tau_{2} / 2$. Echoes of the I-S transitions: (d) $\tau_{4}=\tau_{2}$, (e) $\tau_{4}=2 \tau_{2}$, (f) $\tau_{4}=3 \tau_{2}$. The interpulse delay $\tau_{2}$ should be much shorter than the duration of the FID in order to detect all the echo signals.

The spin-echo sequence considered is represented in Fig. 3, where

$\omega_{\mathrm{Q}}=\frac{3 e^{2} q Q}{8 I(2 I-1) \hbar}\left[3 \cos ^{2} \beta-1+\eta \sin ^{2} \beta \cos 2 \alpha\right]$,

$H_{\mathrm{Q}}^{(1)}=\frac{1}{3} \omega_{\mathrm{Q}}\left[3 I_{z}^{2}-I(I+1)\right]$,

$H_{\mathrm{RF}}=\omega_{\mathrm{RF}} I_{x}$.

The Euler angles $\alpha$ and $\beta$ describe the orientation of the strong static magnetic field in the principal-axis system of the EFG tensor. For numerical applications, $\omega_{\mathrm{RF}} / 2 \pi=50 \mathrm{kHz}$; the first pulse length $t_{1}$ takes three values: 5,3 , or $1 \mu \mathrm{s}$, according as $\omega_{\mathrm{Q}} / 2 \pi=0,50 \mathrm{kHz}$, or $1 \mathrm{MHz}$, respectively. Due to the choice of two $-x$ pulses, line intensities and echo amplitudes have a sign opposite to those of Solomon [1, 2], or Weisman and Bennett [5].

As the first pulse length has an important effect on the echo amplitudes [1], we begin our investigation by analyzing the behavior of the "line intensities" $\left\langle I_{k}^{m, n}\right\rangle$ developed at the end of the first pulse,

$$
\left\langle I_{k}^{m, n}\right\rangle=\operatorname{Tr}\left[\varrho\left(t_{1}\right) I_{k}^{m, n}\right] .
$$

The symbols $I_{k}^{m, n}$ are the fictitious spin- $1 / 2$ operators [9]. In the past $[1,2,4,5]$, the initial state of the spin system, taken at the end of the first pulse, is simply described by the operator $I_{x}$. Table 2 in [10] shows, in 
fact, that this state is more complex if $H_{\mathrm{Q}}^{(1)}$ is taken into account during the pulse. For simplicity, we divide the magnitude of $\omega_{\mathrm{Q}}$ relative to that of $\omega_{\mathrm{RF}}$ into three parts: weak $\left(\omega_{\mathrm{Q}} \ll \omega_{\mathrm{RF}}\right)$, medium $\left(\omega_{\mathrm{Q}} \approx \omega_{\mathrm{RF}}\right)$, and strong $\left(\omega_{\mathrm{Q}} \gg \omega_{\mathrm{RF}}\right)$. As a rule [11], in usual experimental conditions, the line intensity of polarizations $\left(\left\langle I_{z}^{2,5}\right\rangle,\left\langle I_{z}^{1,6}\right\rangle,\left\langle I_{z}^{3,4}\right\rangle\right)$ and that of $1 \mathrm{Q}$ on-resonance coherence $\left\langle I_{y}^{3,4}\right\rangle$ are important whatever the $\omega_{\mathrm{Q}} / \omega_{\mathrm{RF}}$ ratio is. The line intensity of MQ on-resonance coherences $\left(\left\langle I_{y}^{2,5}\right\rangle,\left\langle I_{y}^{1,6}\right\rangle\right)$, as well as that of the $x$ - and $y$-components of MQ off-resonance coherences, are only important for medium $\omega_{\mathrm{Q}}$. For $1 \mathrm{Q}$ off-resonance coherences, the line intensities of their $y$-component, $\left(\left\langle I_{y}^{1,2}\right\rangle,\left\langle I_{y}^{2,3}\right\rangle\right)$, are important for weak and medium $\omega_{\mathrm{Q}}$; on the other hand, their $x$-components are important only for medium $\omega_{\mathrm{Q}}$. Another property is worth noticing: some line intensities remain unchanged when the $-x$ pulse is changed to an $x$ pulse. This is the case for polarizations, $2 \mathrm{Q}$ and $4 \mathrm{Q}$ coherences. Only the line intensities of odd-quantum coherences change sign with that of the pulse [12].

Formally, the study of echoes requires the calculation of the density matrix $\varrho\left(t_{1}, \tau_{2}, t_{3}, \tau_{4}\right)$ describing the spin system during acquisition time,

$$
\varrho\left(t_{1}, \tau_{2}, t_{3}, \tau_{4}\right)=\exp \left(-i H_{\mathrm{Q}}^{(1)} \tau_{4}\right) \varrho\left(t_{1}, \tau_{2}, t_{3}\right) \exp \left(i H_{\mathrm{Q}}^{(1)} \tau_{4}\right) .
$$

In fact, only the components of $\varrho\left(t_{1}, \tau_{2}, t_{3}, \tau_{4}\right)$ associated with $1 \mathrm{Q}$ coherences are required, namely

$$
\begin{aligned}
& \varrho_{4,3}\left(t_{1}, \tau_{2}, t_{3}, \tau_{4}\right)=\exp \left[-i \tau_{4}\left(-8 \omega_{\mathrm{Q}}\right) / 3\right] \varrho_{4,3}\left(t_{1}, \tau_{2}, t_{3}\right) \exp \left[i \tau_{4}\left(-8 \omega_{\mathrm{Q}}\right) / 3\right]=\varrho_{4,3}\left(t_{1}, \tau_{2}, t_{3}\right), \\
& \varrho_{3,2}\left(t_{1}, \tau_{2}, t_{3}, \tau_{4}\right)=\exp \left[-i \tau_{4}\left(-8 \omega_{\mathrm{Q}}\right) / 3\right] \varrho_{3,2}\left(t_{1}, \tau_{2}, t_{3}\right) \exp \left[i \tau_{4}\left(-2 \omega_{\mathrm{Q}}\right) / 3\right]=\varrho_{3,2}\left(t_{1}, \tau_{2}, t_{3}\right) \exp \left(i \tau_{4} 2 \omega_{\mathrm{Q}}\right), \\
& \varrho_{2,1}\left(t_{1}, \tau_{2}, t_{3}, \tau_{4}\right)=\exp \left[-i \tau_{4}\left(-2 \omega_{\mathrm{Q}}\right) / 3\right] \varrho_{2,1}\left(t_{1}, \tau_{2}, t_{3}\right) \exp \left[i \tau_{4}\left(10 \omega_{\mathrm{Q}}\right) / 3\right]=\varrho_{2,1}\left(t_{1}, \tau_{2}, t_{3}\right) \exp \left(i \tau_{4} 4 \omega_{\mathrm{Q}}\right) .
\end{aligned}
$$

The three components $\varrho_{4,3}\left(t_{1}, \tau_{2}, t_{3}\right), \varrho_{3,2}\left(t_{1}, \tau_{2}, t_{3}\right)$ and $\varrho_{2,1}\left(t_{1}, \tau_{2}, t_{3}\right)$ of the density matrix $\varrho\left(t_{1}, \tau_{2}, t_{3}\right)$ at the end of the second pulse were calculated previously [10]. During the acquisition period, $F_{y}^{3,4}\left(t_{1}, \tau_{2}, t_{3}\right)$, $F_{y}^{2,3}\left(t_{1}, \tau_{2}, t_{3}, \tau_{4}\right), F_{x}^{2,3}\left(t_{1}, \tau_{2}, t_{3}, \tau_{4}\right), F_{y}^{1,2}\left(t_{1}, \tau_{2}, t_{3}, \tau_{4}\right)$, and $F_{x}^{1,2}\left(t_{1}, \tau_{2}, t_{3}, \tau_{4}\right)$, the relative intensity of the central line, the $y$ - and $x$ - components of an I-S, and those of an O-S, respectively, are defined by

$$
\begin{aligned}
& i F_{y}^{3,4}\left(t_{1}, \tau_{2}, t_{3}, \tau_{4}\right)=\frac{6}{35} \varrho_{4,3}\left(t_{1}, \tau_{2}, t_{3}, \tau_{4}\right)=\frac{6}{35} \varrho_{4,3}\left(t_{1}, \tau_{2}, t_{3}\right), \\
& F_{x}^{2,3}\left(t_{1}, \tau_{2}, t_{3}, \tau_{4}\right)+i F_{y}^{2,3}\left(t_{1}, \tau_{2}, t_{3}, \tau_{4}\right)=\frac{2 \sqrt{8}}{35} \varrho_{3,2}\left(t_{1}, \tau_{2}, t_{3}, \tau_{4}\right), \\
& F_{x}^{1,2}\left(t_{1}, \tau_{2}, t_{3}, \tau_{4}\right)+i F_{y}^{1,2}\left(t_{1}, \tau_{2}, t_{3}, \tau_{4}\right)=\frac{2 \sqrt{5}}{35} \varrho_{2,1}\left(t_{1}, \tau_{2}, t_{3}, \tau_{4}\right) .
\end{aligned}
$$

Equation (7a) shows that the central line intensity does not depend on $\tau_{4}$. This means than an echo signal cannot be predicted for the central transition. From a practical point of view we must choose an interpulse delay $\tau_{2}$ much shorter than the duration $T_{\text {FID }}$ of the central transition (see Figure 3). This experimental condition has already been mentioned for spin-3/2 systems [13]. Developing (7b) and (7c) with software Mathematica yields lengthy expressions. Only those related to echoes are given in the following. The $x$ - and $y$-components of the echoes detected as I-S signals have the relative amplitudes $\Psi_{x}^{2,3}\left(t_{1}, \tau_{2}, t_{3}, \tau_{4}\right)$ and $\Psi_{y}^{2,3}\left(t_{1}, \tau_{2}, t_{3}, \tau_{4}\right)$, respectively:

$$
\begin{gathered}
\Psi_{x}^{2,3}\left(t_{1}, \tau_{2}, t_{3}, \tau_{4}\right)+i \Psi_{y}^{2,3}\left(t_{1}, \tau_{2}, t_{3}, \tau_{4}\right)=\frac{2 \sqrt{8}}{35}\{[ \\
\left.+\sin 2 \omega_{\mathrm{Q}}\left(\tau_{4}-\tau_{2}\right)-i \cos 2 \omega_{\mathrm{Q}}\left(\tau_{4}-\tau_{2}\right)\right]\left[\left\langle I_{y}^{2,3}\right\rangle C_{1}+\left\langle I_{x}^{2,4}\right\rangle C_{4}\right] \\
+\left[\cos 2 \omega_{\mathrm{Q}}\left(\tau_{4}-\tau_{2}\right)+i \sin 2 \omega_{\mathrm{Q}}\left(\tau_{4}-\tau_{2}\right)\right]\left[-\left\langle I_{y}^{2,4}\right\rangle C_{4}+\left\langle I_{x}^{2,3}\right\rangle C_{1}\right] \\
+\frac{1}{2}\left[\cos 2 \omega_{\mathrm{Q}}\left(\tau_{4}-2 \tau_{2}\right)+i \sin 2 \omega_{\mathrm{Q}}\left(\tau_{4}-2 \tau_{2}\right)\right][ \\
-\left(\left\langle I_{y}^{1,2}\right\rangle+\left\langle I_{y}^{1,5}\right\rangle\right) G_{6}+\left(\left\langle I_{y}^{1,2}\right\rangle-\left\langle I_{y}^{1,5}\right\rangle\right) G_{4} \\
\left.+\left(\left\langle I_{x}^{1,2}\right\rangle+\left\langle I_{x}^{1,5}\right\rangle\right) G_{5}+\left(\left\langle I_{x}^{1,2}\right\rangle-\left\langle I_{x}^{1,5}\right\rangle\right) G_{3}\right] \\
+\frac{1}{2}\left[\sin 2 \omega_{\mathrm{Q}}\left(\tau_{4}-2 \tau_{2}\right)-i \cos 2 \omega_{\mathrm{Q}}\left(\tau_{4}-2 \tau_{2}\right)\right]\left[\left(\left\langle I_{y}^{1,2}\right\rangle+\left\langle I_{y}^{1,5}\right\rangle\right) G_{5}+\left(\left\langle I_{y}^{1,2}\right\rangle-\left\langle I_{y}^{1,5}\right\rangle\right) G_{3}\right. \\
\left.+\left(\left\langle I_{x}^{1,2}\right\rangle+\left\langle I_{x}^{1,5}\right\rangle\right) G_{6}-\left(\left\langle I_{x}^{1,2}\right\rangle-\left\langle I_{x}^{1,5}\right\rangle\right) G_{4}\right]
\end{gathered}
$$




$$
\begin{array}{r}
+\frac{1}{2}\left[\cos 2 \omega_{\mathrm{Q}}\left(\tau_{4}-3 \tau_{2}\right)+i \sin 2 \omega_{\mathrm{Q}}\left(\tau_{4}-3 \tau_{2}\right)\right]\left[-\left(\left\langle I_{y}^{1,3}\right\rangle+\left\langle I_{y}^{1,4}\right\rangle\right) C_{12}+\left(\left\langle I_{y}^{1,3}\right\rangle-\left\langle I_{y}^{1,4}\right\rangle\right) C_{14}\right. \\
\left.+\left(\left\langle I_{x}^{1,3}\right\rangle+\left\langle I_{x}^{1,4}\right\rangle\right) C_{11}+\left(\left\langle I_{x}^{1,3}\right\rangle-\left\langle I_{x}^{1,4}\right\rangle\right) C_{13}\right] \\
+\frac{1}{2}\left[\sin 2 \omega_{\mathrm{Q}}\left(\tau_{4}-3 \tau_{2}\right)-i \cos 2 \omega_{\mathrm{Q}}\left(\tau_{4}-3 \tau_{2}\right)\right]\left[\left(\left\langle I_{y}^{1,3}\right\rangle+\left\langle I_{y}^{1,4}\right\rangle\right) C_{11}+\left(\left\langle I_{y}^{1,3}\right\rangle-\left\langle I_{y}^{1,4}\right\rangle\right) C_{13}\right. \\
\left.\left.+\left(\left\langle I_{x}^{1,3}\right\rangle+\left\langle I_{x}^{1,4}\right\rangle\right) C_{12}-\left(\left\langle I_{x}^{1,3}\right\rangle-\left\langle I_{x}^{1,4}\right\rangle\right) C_{14}\right]\right\} .
\end{array}
$$

The functions $G_{i}$, defined in Table 1, depend on the second pulse length $t_{3}$. The $x$ - and $y$-components of the echoes detected as O-S signals have the relative amplitudes $\Psi_{x}^{1,2}\left(t_{1}, \tau_{2}, t_{3}, \tau_{4}\right)$ and $\Psi_{y}^{1,2}\left(t_{1}, \tau_{2}, t_{3}, \tau_{4}\right)$ given by

$$
\begin{gathered}
\Psi_{x}^{1,2}\left(t_{1}, \tau_{2}, t_{3}, \tau_{4}\right)+i \Psi_{y}^{1,2}\left(t_{1}, \tau_{2}, t_{3}, \tau_{4}\right)=\frac{2 \sqrt{5}}{35}\left\{\left[\sin 4 \omega_{\mathrm{Q}}\left(\tau_{4}-\tau_{2}\right)-i \cos 4 \omega_{\mathrm{Q}}\left(\tau_{4}-\tau_{2}\right)\right]\left[\left\langle I_{y}^{1,2}\right\rangle G_{1}+\left\langle I_{x}^{1,5}\right\rangle G_{2}\right]\right. \\
+\left[\cos 4 \omega_{\mathrm{Q}}\left(\tau_{4}-\tau_{2}\right)+i \sin 4 \omega_{\mathrm{Q}}\left(\tau_{4}-\tau_{2}\right)\right]\left[-\left\langle I_{y}^{1,5}\right\rangle G_{2}+\left\langle I_{x}^{1,2}\right\rangle G_{1}\right] \\
+\frac{1}{2}\left[\sin 4 \omega_{\mathrm{Q}}\left(\tau_{4}-\tau_{2} / 2\right)-i \cos 4 \omega_{\mathrm{Q}}\left(\tau_{4}-\tau_{2} / 2\right)\right]\left[\left(\left\langle I_{y}^{2,3}\right\rangle+\left\langle I_{y}^{2,4}\right\rangle\right) G_{3}+\left(\left\langle I_{y}^{2,3}\right\rangle-\left\langle I_{y}^{2,4}\right\rangle\right) G_{5}\right. \\
\left.+\left(\left\langle I_{x}^{2,3}\right\rangle+\left\langle I_{x}^{2,4}\right\rangle\right) G_{4}-\left(\left\langle I_{x}^{2,3}\right\rangle-\left\langle I_{x}^{2,4}\right\rangle\right) G_{6}\right] \\
+\frac{1}{2}\left[\cos 4 \omega_{\mathrm{Q}}\left(\tau_{4}-\tau_{2} / 2\right)+i \sin 4 \omega_{\mathrm{Q}}\left(\tau_{4}-\tau_{2} / 2\right)\right]\left[-\left(\left\langle I_{y}^{2,3}\right\rangle+\left\langle I_{y}^{2,4}\right\rangle\right) G_{4}+\left(\left\langle I_{y}^{2,3}\right\rangle-\left\langle I_{y}^{2,4}\right\rangle\right) G_{6}\right. \\
\left.+\left(\left\langle I_{x}^{2,3}\right\rangle+\left\langle I_{x}^{2,4}\right\rangle\right) G_{3}+\left(\left\langle I_{x}^{2,3}\right\rangle-\left\langle I_{x}^{2,4}\right\rangle\right) G_{5}\right] \\
+\frac{1}{2}\left[\sin 4 \omega_{\mathrm{Q}}\left(\tau_{4}-3 \tau_{2} / 2\right)-i \cos 4 \omega_{\mathrm{Q}}\left(\tau_{4}-3 \tau_{2} / 2\right)\right]\left[\left(\left\langle I_{y}^{1,3}\right\rangle+\left\langle I_{y}^{1,4}\right\rangle\right) G_{7}+\left(\left\langle I_{y}^{1,3}\right\rangle-\left\langle I_{y}^{1,4}\right\rangle\right) G_{9}\right. \\
\left.+\left(\left\langle I_{x}^{1,3}\right\rangle+\left\langle I_{x}^{1,4}\right\rangle\right) G_{8}-\left(\left\langle I_{x}^{1,3}\right\rangle-\left\langle I_{x}^{1,4}\right\rangle\right) G_{10}\right] \\
+\frac{1}{2}\left[\cos 4 \omega_{\mathrm{Q}}\left(\tau_{4}-3 \tau_{2} / 2\right)+i \sin 4 \omega_{\mathrm{Q}}\left(\tau_{4}-3 \tau_{2} / 2\right)\right]\left[-\left(\left\langle I_{y}^{1,3}\right\rangle+\left\langle I_{y}^{1,4}\right\rangle\right) G_{8}+\left(\left\langle I_{y}^{1,3}\right\rangle-\left\langle I_{y}^{1,4}\right\rangle\right) G_{10}\right. \\
\left.\left.+\left(\left\langle I_{x}^{1,3}\right\rangle+\left\langle I_{x}^{1,4}\right\rangle\right) G_{7}+\left(\left\langle I_{x}^{1,3}\right\rangle-\left\langle I_{x}^{1,4}\right\rangle\right) G_{9}\right]\right\} .
\end{gathered}
$$

The angular velocity term in the two trigonometric functions, sine and cosine, is $2 \omega_{\mathrm{Q}}$ for I-S echoes, (8), and $4 \omega_{\mathrm{Q}}$ for O-S echoes, (9). In other words, the I-S echoes are twice as broad as the O-S ones.

In quadrature detection mode, (8) and (9) reveal six echoes in both channels. Three echoes as I-S signals are located at $\tau_{4}=\tau_{2}, \tau_{4}=2 \tau_{2}$ and $\tau_{4}=3 \tau_{2}$. Three other as O-S signals are located at $\tau_{4}=\tau_{2} / 2, \tau_{4}=\tau_{2}$ and $\tau_{4}=3 \tau_{2} / 2$. These echoes are due to the refocusing of off-resonance coherences developed during the first pulse. In fact, each echo is the sum of two components: one has a symmetrical bell shape related to the cosine functions, the other has a sine-like shape connected to the sine functions. The sine-like shaped echoes do not modify the maximum of the bell shaped echo amplitude, because they are always zero at these positions,

$G_{1}\left(t_{3}\right)=X_{m+} Y_{m+} Y_{j-} Z_{j-} \cos \omega_{m j} t_{3}$ $G_{2}\left(t_{3}\right)=X_{m+} Y_{m+} Y_{j-} Z_{j-} \sin \omega_{m j} t_{3}$ $G_{3}\left(t_{3}\right)=Y_{m+} Y_{m+} X_{j-} Z_{j-} \cos \omega_{m j} t_{3}$ $G_{4}\left(t_{3}\right)=Y_{m+} Y_{m+} X_{j-} Z_{j-} \sin \omega_{m j} t_{3}$ $G_{5}\left(t_{3}\right)=X_{m+} Z_{m+} Y_{j-} Y_{j-} \cos \omega_{m j} t_{3}$ $G_{6}\left(t_{3}\right)=X_{m+} Z_{m+} Y_{j-} Y_{j-} \sin \omega_{m j} t_{3}$ $G_{7}\left(t_{3}\right)=X_{m+} Y_{m+} X_{j-} Z_{j-} \cos \omega_{m j} t_{3}$ $G_{8}\left(t_{3}\right)=X_{m+} Y_{m+} X_{j-} Z_{j-} \sin \omega_{m j} t_{3}$ $G_{9}\left(t_{3}\right)=X_{m+} Z_{m+} Y_{j-} Z_{j-} \cos \omega_{m j} t_{3}$ $G_{10}\left(t_{3}\right)=X_{m+} Z_{m+} Y_{j-} Z_{j-} \sin \omega_{m j} t_{3}$$$
G_{i}\left(t_{3}\right) \text {. For clarity, }
$$$$
\text { in front of each term }
$$$$
\text { has been omitted. }
$$

but alter their shapes. As a result, the echoes can take any shape ranging from a symmetrical bell to an asymmetric sine-like shape, depending on the experimental conditions.

As shown previously for spin-3/2 systems [11], measurement of echo amplitude versus second pulse length enables us to determine $\omega_{\mathrm{Q}}$. Therefore, in the following, we analyze the amplitude of the six bell shaped echoes in the two detection channels. They are presented for increasing values of $\tau_{4}$.

The relative amplitudes of the first echo located at $\tau_{4}=\tau_{2} / 2$ are given by

$$
\begin{aligned}
& E_{y}^{1,2}\left(t_{1}, t_{3}, \tau_{4}=\tau_{2} / 2\right) \\
& =-\frac{\sqrt{5}}{35}\left[\left(\left\langle I_{y}^{2,3}\right\rangle+\left\langle I_{y}^{2,4}\right\rangle\right) G_{3}+\left(\left\langle I_{y}^{2,3}\right\rangle-\left\langle I_{y}^{2,4}\right\rangle\right) G_{5}\right. \\
& \left.\quad+\left(\left\langle I_{x}^{2,3}\right\rangle+\left\langle I_{x}^{2,4}\right\rangle\right) G_{4}-\left(\left\langle I_{x}^{2,3}\right\rangle-\left\langle I_{x}^{2,4}\right\rangle\right) G_{6}\right], \\
& E_{x}^{1,2}\left(t_{1}, t_{3}, \tau_{4}=\tau_{2} / 2\right) \\
& =+\frac{\sqrt{5}}{35}\left[-\left(\left\langle I_{y}^{2,3}\right\rangle+\left\langle I_{y}^{2,4}\right\rangle\right) G_{4}+\left(\left\langle I_{y}^{2,3}\right\rangle-\left\langle I_{y}^{2,4}\right\rangle\right) G_{6}\right. \\
& \left.+\left(\left\langle I_{x}^{2,3}\right\rangle+\left\langle I_{x}^{2,4}\right\rangle\right) G_{3}+\left(\left\langle I_{x}^{2,3}\right\rangle-\left\langle I_{x}^{2,4}\right\rangle\right) G_{5}\right]
\end{aligned}
$$$$
\text { the symbol } \sum_{m=1}^{3} \sum_{j=1}^{3} \quad E_{x}^{1,2}\left(t_{1}, t_{3}, \tau_{4}=\tau_{2} / 2\right)
$$

this echo is the refocusing of I-S $(2 \leftrightarrow 3)$ and $2 \mathrm{Q}$ $(2 \leftrightarrow 4)$ coherences. The graphs of $E_{y}^{1,2}\left(t_{1}, t_{3}, \tau_{4}=\tau_{2} / 2\right)$ 


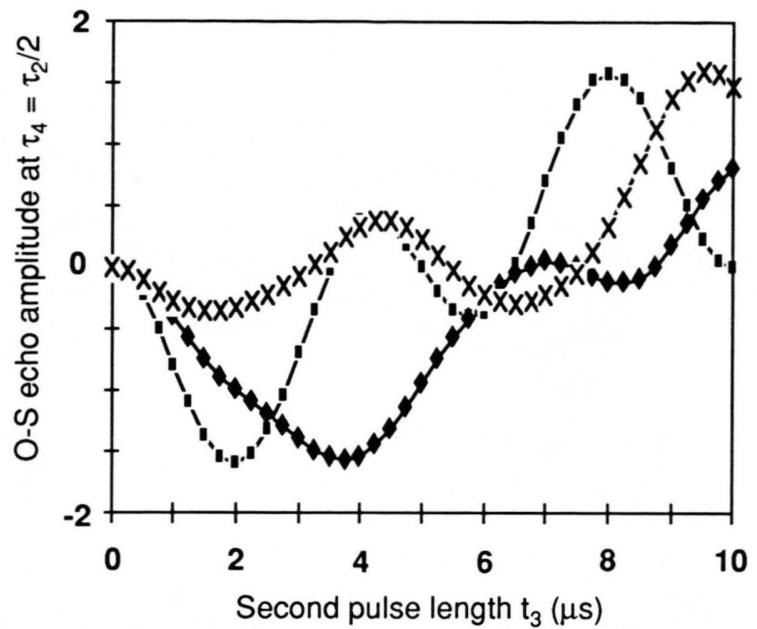

Fig. 4. Theoretical echo amplitude $E_{y}^{1,2}\left(t_{1}, t_{3}, \tau_{4}=\tau_{2} / 2\right)$, (10a), versus $t_{3}$ for two $\omega_{\mathrm{O}} / 2 \pi$ values: $(\bullet) 0 \mathrm{kHz},(\bullet) 50 \mathrm{kHz}$. (x) Theoretical echo amplitude $E_{x}^{1,2}\left(t_{1}=3 \mu \mathrm{s}, t_{3}, \tau_{4}=\tau_{2} / 2\right)$, (10b), for $\omega_{\mathrm{Q}} / 2 \pi=50 \mathrm{kHz}$.

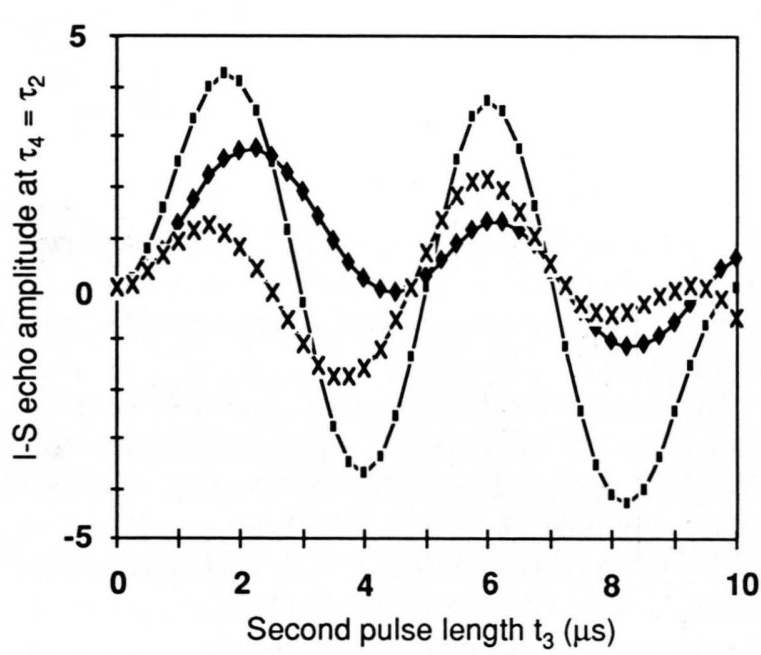

Fig. 5. Theoretical echo amplitude $E_{y}^{2,3}\left(t_{1}, t_{3}, \tau_{4}=\tau_{2}\right),(11 \mathrm{a})$, versus $t_{3}$ for two $\omega_{\mathrm{Q}} / 2 \pi$ values: $(\bullet) 0 \mathrm{kHz},(\bullet) 50 \mathrm{kHz}$. $(\times)$ Theoretical echo amplitude $E_{x}^{2,3}\left(t_{1}=3 \mu \mathrm{s}, t_{3}, \tau_{4}=\tau_{2}\right),(11 \mathrm{~b})$, for $\omega_{\mathrm{Q}} / 2 \pi=50 \mathrm{kHz}$.

and $E_{x}^{1,2}\left(t_{1}, t_{3}, \tau_{4}=\tau_{2} / 2\right)$ versus the second pulse length $t_{3}$ are represented in Figure 4.

At $\tau_{4}=\tau_{2}$, two overlaping echoes are detected. Fortunately, their widths are different as mentioned above. The relative amplitudes of the I-S one are given by

$E_{y}^{2,3}\left(t_{1}, t_{3}, \tau_{4}=\tau_{2}\right)=-\frac{2 \sqrt{8}}{35}\left(\left\langle I_{y}^{2,3}\right\rangle C_{1}+\left\langle I_{y}^{2,4}\right\rangle C_{4}\right)$

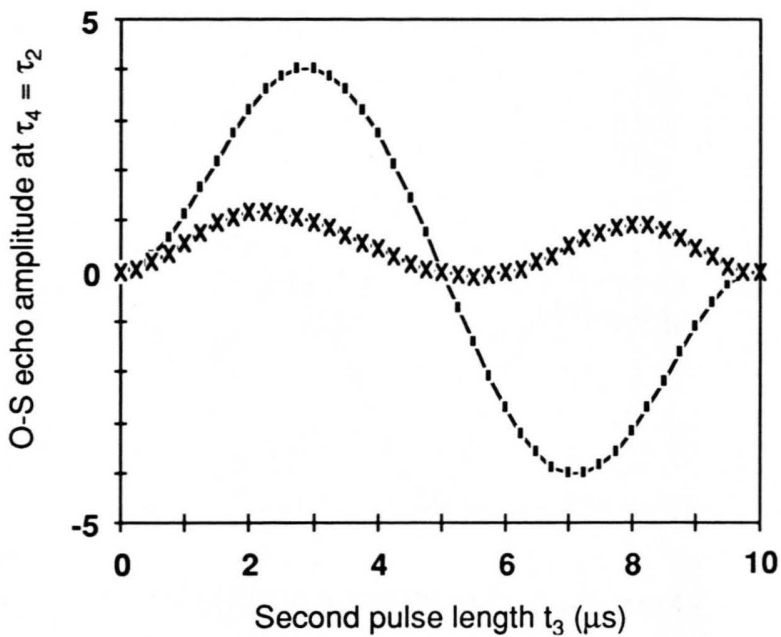

Fig. 6. Theoretical echo amplitude versus $t_{3}:(\varpi) E_{y}^{1,2}$ $\left(t_{1}=5 \mu \mathrm{s}, t_{3}, \tau_{4}=\tau_{2}\right),(12 \mathrm{a})$, for $\omega_{\mathrm{Q}} / 2 \pi=0 \mathrm{kHz},(\times) E_{x}^{1,2}$ $\left(t_{1}=3 \mu \mathrm{s}, t_{3}, \tau_{4}=\tau_{2}\right),(12 \mathrm{~b})$, for $\omega_{\mathrm{Q}} / 2 \pi=50 \mathrm{kHz}$.

$E_{x}^{2,3}\left(t_{1}, t_{3}, \tau_{4}=\tau_{2}\right)=\frac{2 \sqrt{8}}{35}\left(-\left\langle I_{y}^{2,4}\right\rangle C_{4}+\left\langle I_{y}^{2,3}\right\rangle C_{1}\right)$,

this echo is the refocusing of I-S $(2 \leftrightarrow 3)$ and $2 \mathrm{Q}(2 \leftrightarrow 4)$ coherences. Figure 5 shows the graphs of $E_{y}^{2,3}\left(t_{1}, t_{3}, \tau_{4}=\tau_{2}\right)$ and $E_{x}^{2,3}\left(t_{1}, t_{3}, \tau_{4}=\tau_{2}\right)$ versus $t_{3}$. The relative amplitudes of the O-S echo are given by

$E_{y}^{1,2}\left(t_{1}, t_{3}, \tau_{4}=\tau_{2}\right)=-\frac{2 \sqrt{5}}{35}\left(\left\langle I_{y}^{1,2}\right\rangle G_{1}+\left\langle I_{x}^{1,5}\right\rangle G_{2}\right)$,

$E_{x}^{1,2}\left(t_{1}, t_{3}, \tau_{4}=\tau_{2}\right)=\frac{2 \sqrt{5}}{35}\left(-\left\langle I_{y}^{1,5}\right\rangle G_{2}+\left\langle I_{x}^{1,2}\right\rangle G_{1}\right) ;$

this echo is the refocusing of O-S $(1 \leftrightarrow 2)$ and $4 \mathrm{Q}$ $(1 \leftrightarrow 5)$ coherences. Figure 6 represents the graphs of $E_{y}^{1,2}\left(t_{1}, t_{3}, \tau_{4}=\tau_{2}\right)$ and $E_{x}^{1,2}\left(t_{1}, t_{3}, \tau_{4}=\tau_{2}\right)$ versus $t_{3}$.

The relative amplitudes of the fourth echo, located at $\tau_{4}=3 \tau_{2} / 2$, are given by

$$
\begin{gathered}
E_{y}^{1,2}\left(t_{1}, t_{3}, \tau_{4}=3 \tau_{2} / 2\right) \\
=-\frac{\sqrt{5}}{35}\left[\left(\left\langle I_{y}^{1,3}\right\rangle+\left\langle I_{y}^{1,4}\right\rangle\right) G_{7}+\left(\left\langle I_{y}^{1,3}\right\rangle-\left\langle I_{y}^{1,4}\right\rangle\right) G_{9}\right. \\
\left.\quad+\left(\left\langle I_{x}^{1,3}\right\rangle+\left\langle I_{x}^{1,4}\right\rangle\right) G_{8}-\left(\left\langle I_{x}^{1,3}\right\rangle-\left\langle I_{x}^{1,4}\right\rangle\right) G_{10}\right], \\
E_{x}^{1,2}\left(t_{1}, t_{3}, \tau_{4}=3 \tau_{2} / 2\right) \\
=\frac{\sqrt{5}}{35}\left[-\left(\left\langle I_{y}^{1,3}\right\rangle+\left\langle I_{y}^{1,4}\right\rangle\right) G_{8}+\left(\left\langle I_{y}^{1,3}\right\rangle-\left\langle I_{y}^{1,4}\right\rangle\right) G_{10}\right. \\
\left.\quad+\left(\left\langle I_{x}^{1,3}\right\rangle+\left\langle I_{x}^{1,4}\right\rangle\right) G_{7}+\left(\left\langle I_{x}^{1,3}\right\rangle-\left\langle I_{x}^{1,4}\right\rangle\right) G_{9}\right]
\end{gathered}
$$




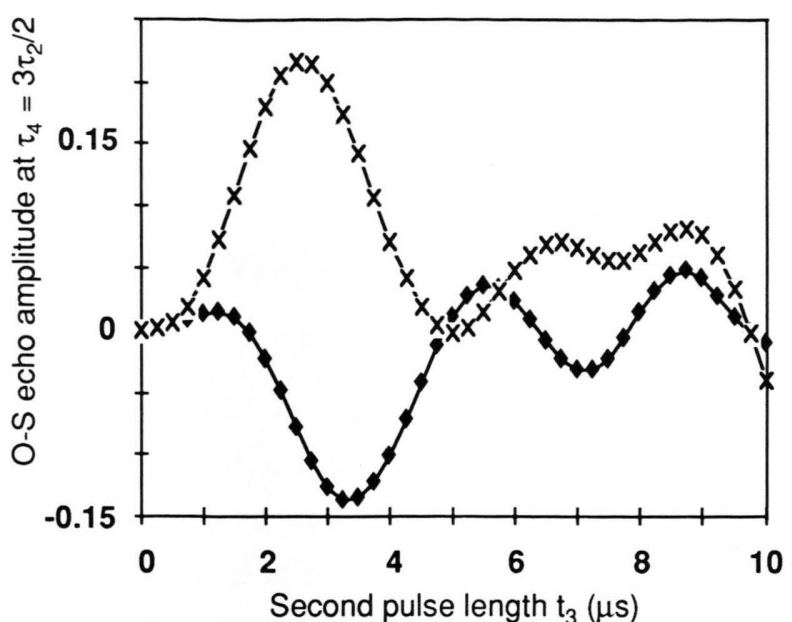

Fig. 7. Theoretical echo amplitude versus $t_{3}$ for $\omega_{0} /$ $2 \pi=50 \mathrm{kHz}:(\bullet) E_{y}^{1,2}\left(t_{1}=3 \mu \mathrm{s}, t_{3}, \tau_{4}=3 \tau_{2} / 2\right) .(13 \mathrm{a}),(\times) E_{x}^{1,2}$ $\left(t_{1}=3 \mu \mathrm{s}, t_{3}, \tau_{4}=3 \tau_{2} / 2\right)$, (13b).

this echo is the refocusing of $2 \mathrm{Q}(1 \leftrightarrow 3)$ and $3 \mathrm{Q}$ $(1 \leftrightarrow 4)$ coherences. The graphs of $E_{y}^{1,2}\left(t_{1}, t_{3}, \tau_{4}=\right.$ $\left.3 \tau_{2} / 2\right)$ and $E_{x}^{1,2}\left(t_{1}, t_{3}, \tau_{4}=3 \tau_{2} / 2\right)$ versus $t_{3}$ are depicted in Figure 7.

The fifth echo is located at $\tau_{4}=2 \tau_{2}$. Its relative amplitudes are given by

$$
\begin{aligned}
E_{y}^{2,3}( & \left.t_{1}, t_{3}, \tau_{4}=2 \tau_{2}\right) \\
= & -\frac{\sqrt{8}}{35}\left[\left(\left\langle I_{y}^{1,2}\right\rangle+\left\langle I_{y}^{1,5}\right\rangle\right) G_{5}+\left(\left\langle I_{y}^{1,2}\right\rangle-\left\langle I_{y}^{1,5}\right\rangle\right) G_{3}\right. \\
& \left.+\left(\left\langle I_{x}^{1,2}\right\rangle+\left\langle I_{x}^{1,5}\right\rangle\right) G_{6}-\left(\left\langle I_{x}^{1,2}\right\rangle-\left\langle I_{x}^{1,5}\right\rangle\right) G_{4}\right], \\
E_{x}^{2,3}\left(t_{1}, t_{3}, \tau_{4}=2 \tau_{2}\right) & \\
= & \frac{\sqrt{8}}{35}\left[-\left(\left\langle I_{y}^{1,2}\right\rangle+\left\langle I_{y}^{1,5}\right\rangle\right) G_{6}+\left(\left\langle I_{y}^{1,2}\right\rangle-\left\langle I_{y}^{1,5}\right\rangle\right) G_{4}\right. \\
+ & \left.\left(\left\langle I_{x}^{1,2}\right\rangle+\left\langle I_{x}^{1,5}\right\rangle\right) G_{5}+\left(\left\langle I_{x}^{1,2}\right\rangle-\left\langle I_{x}^{1,5}\right\rangle\right) G_{3}\right]
\end{aligned}
$$

this echo is the refocusing of the $\mathrm{O}-\mathrm{S}(1 \leftrightarrow 2)$ and $4 \mathrm{Q}(1 \leftrightarrow 5)$ coherences. The graphs of $\mathrm{E}_{y}^{2,3}$ $\left(t_{1}, t_{3}, \tau_{4}=2 \tau_{2}\right)$ and $E_{x}^{2,3}\left(t_{1}, t_{3}, \tau_{4}=2 \tau_{2}\right)$ against $t_{3}$ are plotted in Figure 8.

Finally, at $\tau_{4}=3 \tau_{2}$ is located the last echo, whose relative amplitudes are given by

$$
\begin{aligned}
& E_{y}^{2,3}\left(t_{1}, t_{3}, \tau_{4}=3 \tau_{2}\right) \\
& =-\frac{\sqrt{8}}{35}\left[\left(\left\langle I_{y}^{1,3}\right\rangle+\left\langle I_{y}^{1,4}\right\rangle\right) C_{11}+\left(\left\langle I_{y}^{1,3}\right\rangle-\left\langle I_{y}^{1,4}\right\rangle\right) C_{13}\right. \\
& \left.\quad+\left(\left\langle I_{x}^{1,3}\right\rangle+\left\langle I_{x}^{1,4}\right\rangle\right) C_{12}-\left(\left\langle I_{x}^{1,3}\right\rangle-\left\langle I_{x}^{1,4}\right\rangle\right) C_{14}\right],
\end{aligned}
$$

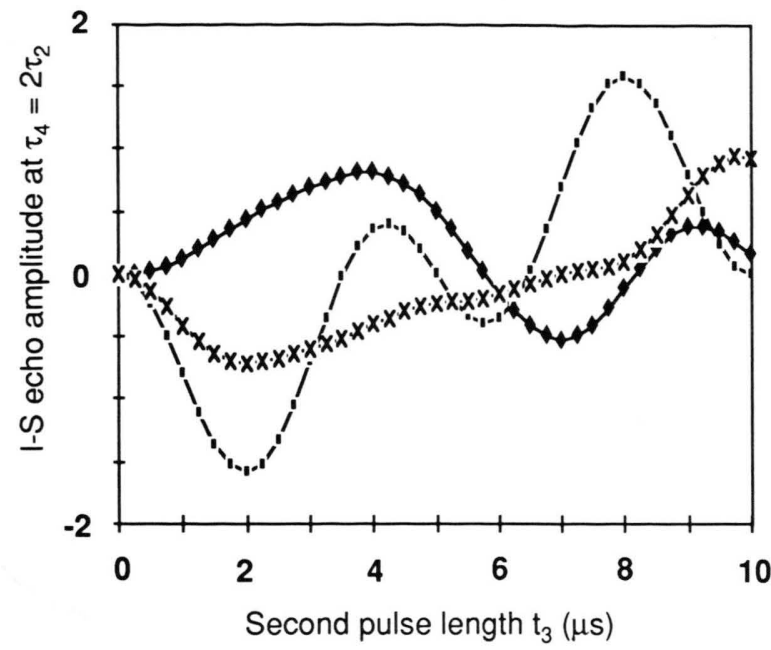

Fig. 8. Theoretical echo amplitude versus $t_{3}$ : (匹) $E_{y}^{2,3}$ $\left(t_{1}=5 \mu \mathrm{s}, t_{3}, \tau_{4}=2 \tau_{2}\right), \omega_{\mathrm{Q}} / 2 \pi=0 \mathrm{kHz},(\bullet) E_{y}^{2,3}\left(t_{1}=3 \mu \mathrm{s}, t_{3}\right.$, $\left.\tau_{4}=2 \tau_{2}\right), \omega_{\mathrm{Q}} / 2 \pi=50 \mathrm{kHz},(14 \mathrm{a}),(\times) E_{x}^{2,3}\left(t_{1}=3 \mu \mathrm{s}, t_{3}, \tau_{4}=\right.$ $\left.2 \tau_{2}\right), \omega_{\mathrm{Q}} / 2 \pi=50 \mathrm{kHz},(14 \mathrm{~b})$.

$$
\begin{aligned}
& E_{x}^{2,3}\left(t_{1}, t_{3}, \tau_{4}=3 \tau_{2}\right) \\
& =\frac{\sqrt{8}}{35}\left[-\left(\left\langle I_{y}^{1,3}\right\rangle+\left\langle I_{y}^{1,4}\right\rangle\right) C_{12}+\left(\left\langle I_{y}^{1,3}\right\rangle-\left\langle I_{y}^{1,4}\right\rangle\right) C_{14}\right. \\
& \left.\quad+\left(\left\langle I_{x}^{1,3}\right\rangle+\left\langle I_{x}^{1,4}\right\rangle\right) C_{11}+\left(\left\langle I_{x}^{1,3}\right\rangle-\left\langle I_{x}^{1,4}\right\rangle\right) C_{13}\right]
\end{aligned}
$$

this echo is the refocusing of $2 \mathrm{Q}(1 \leftrightarrow 3)$ and $3 \mathrm{Q}$ $(1 \leftrightarrow 4)$ coherences. The graphs of $E_{y}^{2,3}\left(t_{1}, t_{3}, \tau_{4}=3 \tau_{2}\right)$ and $E_{x}^{2,3}\left(t_{1}, t_{3}, \tau_{4}=3 \tau_{2}\right)$ versus $t_{3}$ are represented in Figure 9.

The general trend of the echo amplitude deduced from Figs. 4 to 9 is that their magnitude decreases in the following way: I-S echo amplitude at $\tau_{4}=\tau_{2}$, O-S echo amplitude at $\tau_{4}=\tau_{2}$, O-S echo amplitude at $\tau_{4}=\tau_{2} / 2$, I-S echo amplitude at $\tau_{4}=2 \tau_{2}$, I-S echo amplitude at $\tau_{4}=3 \tau_{2}$, O-S echo amplitude at $\tau_{4}=3 \tau_{2} / 2$. In addition, the $x$-components of the echoes are only important for medium $\omega_{0}$. On the other hand, the echo amplitude are sums of terms that are the product of two functions, one depending of $t_{1}$ and the other on $t_{3}$. So, the optimization of the echo amplitudes requires both suitable pulse lengths $t_{1}$ and $t_{3}$.

In the early papers, the forbidden echoes occurring at $\tau_{4}=3 \tau_{2} / 2$ and $\tau=3 \tau_{2}$ are not predicted because they are due to the refocusing of MQ transitions developed during the first pulse. In order to compare with early results (Table 2), we deal with the particuler case of the hard pulse excitation. In this case, the MQ coherence line intensities are negligible, the echo amplitudes are due to $1 \mathrm{Q}$ coherences only and have the 


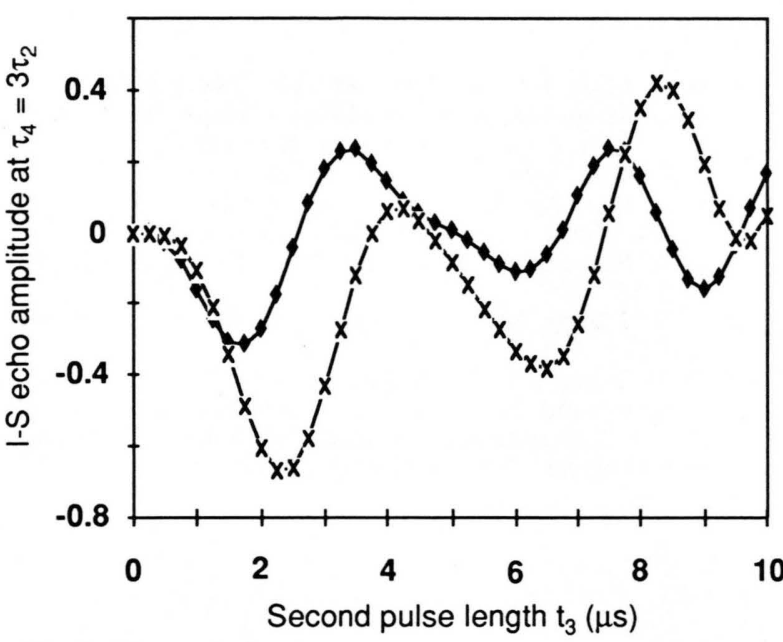

Fig. 9. Theoretical echo amplitude versus $t_{3}$ for $\omega_{0} / 2 \pi=$ $50 \mathrm{kHz}:(\bullet) E_{y}^{2,3}\left(t_{1}=3 \mu \mathrm{s}, t_{3}, \tau_{4}=3 \tau_{2}\right),(15 \mathrm{a}),(\times) E_{x}^{2,3}\left(t_{1}=\right.$ $\left.3 \mu \mathrm{s}, t_{3}, \tau_{4}=3 \tau_{2}\right),(15 \mathrm{~b})$.

following expressions:

$$
\begin{aligned}
& 2 E_{y(0)}^{1,2}\left(t_{1}=t_{90^{\circ}}, t_{3}, \tau_{4}=\tau_{2} / 2\right) \\
& =-\frac{2 \sqrt{5}}{35}\left\langle I_{y}^{2,3}\left(t_{1}=t_{90^{\circ}}\right)\right\rangle\left(G_{3}+G_{5}\right), \\
& \propto-20\left[\cos ^{3} \phi \sin ^{2} \phi\left(\cos ^{5} \phi-4 \cos ^{3} \phi \sin ^{2} \phi\right)\right. \\
& \left.-\cos ^{2} \phi \sin ^{3} \phi\left(\sin ^{5} \phi-4 \cos ^{2} \phi \sin ^{3} \phi\right)\right], \\
& 2 E_{y(0)}^{2,3}\left(t_{1}=t_{90^{\circ}}, t_{3}, \tau_{4}=\tau_{2}\right) \\
& =-\frac{4 \sqrt{8}}{35}\left\langle I_{y}^{2,3}\left(t_{1}=t_{90^{\circ}}\right)\right\rangle C_{1}, \\
& \propto-64\left[\cos ^{2} \phi \sin ^{4} \phi\left(\frac{3}{2} \cos ^{2} \phi-\sin ^{2} \phi\right)^{2}\right. \\
& \left.-\cos ^{4} \phi \sin ^{2} \phi\left(\cos ^{2} \phi-\frac{3}{2} \sin ^{2} \phi\right)^{2}\right], \\
& 2 E_{y(0)}^{1,2}\left(t_{1}=t_{90^{\circ}}, t_{3}, \tau_{4}=\tau_{2}\right) \\
& =-\frac{4 \sqrt{5}}{35}\left\langle I_{y}^{1,2}\left(t_{1}=t_{90^{\circ}}\right)\right\rangle G_{1}, \\
& \propto-25\left[\cos ^{2} \phi \sin ^{8} \phi-\cos ^{8} \phi-\sin ^{2} \phi\right] . \\
& 2 E_{y(0)}^{2,3}\left(t_{1}=t_{90^{\circ}}, t_{3}, \tau_{4}=2 \tau_{2}\right) \\
& =-\frac{2 \sqrt{8}}{35}\left\langle I_{y}^{1,2}\left(t_{1}=t_{90^{\circ}}\right)\right\rangle\left(G_{3}+G_{5}\right), \\
& \propto-20\left[\cos ^{3} \phi \sin ^{2} \phi\left(\cos ^{5} \phi-4 \cos ^{3} \phi \sin ^{2} \phi\right)\right. \\
& \left.-\cos ^{2} \phi \sin ^{3} \phi\left(\sin ^{5} \phi-4 \cos ^{2} \phi \sin ^{3} \phi\right)\right] \text {, } \\
& 2 E_{y(0)}^{1,2}\left(t_{1}=t_{90^{\circ}}, t_{3}, \tau_{4}=3 \tau_{2} / 2\right)=0, \\
& 2 E_{y(0)}^{2,3}\left(t_{1}=t_{90^{\circ}}, t_{3}, \tau_{4}=3 \tau_{2}\right)=0 \text {. }
\end{aligned}
$$

\begin{tabular}{|c|c|c|c|}
\hline \multicolumn{2}{|c|}{ Echo position } & \multirow{2}{*}{$\begin{array}{l}\text { Inner satellite } \\
\text { signals }\end{array}$} & \multirow{2}{*}{$\begin{array}{l}\text { Outer satellite } \\
\text { signals }\end{array}$} \\
\hline this work & early work ${ }^{a}$ & & \\
\hline$\tau_{4}=\tau_{2} / 2$ & $3 \tau / 2$ & & $\begin{array}{l}2 \leftrightarrow 3(1 \mathrm{Q}) \\
2 \leftrightarrow 4(2 \mathrm{Q})\end{array}$ \\
\hline$\tau_{4}=\tau_{2}$ & $2 \tau$ & $\begin{array}{l}2 \leftrightarrow 3(1 \mathrm{Q}) \\
2 \leftrightarrow 4(2 \mathrm{Q})\end{array}$ & $\begin{array}{l}1 \leftrightarrow 2(1 \mathrm{Q}) \\
1 \leftrightarrow 5(4 \mathrm{Q})\end{array}$ \\
\hline$\tau_{4}=3 \tau_{2} / 2$ & & & $\begin{array}{l}1 \leftrightarrow 3(2 Q) \\
1 \leftrightarrow 4(3 Q)\end{array}$ \\
\hline$\tau_{4}=2 \tau_{2}$ & $3 \tau$ & $\begin{array}{l}1 \leftrightarrow 2(1 Q) \\
1 \leftrightarrow 5(4 Q)\end{array}$ & \\
\hline$\tau_{4}=3 \tau_{2}$ & & $\begin{array}{l}1 \leftrightarrow 3(2 \mathrm{Q}) \\
1 \leftrightarrow 4(3 \mathrm{Q})\end{array}$ & \\
\hline
\end{tabular}

Table 2. Coherences ( $1 \mathrm{Q}$ to $4 \mathrm{Q}$ ) developed during the first pulse and refocused as inner and outer satellite echoes.

a In early work, the two RF pulses are separated by an interpulse delay $\tau$ and the time origine starts from the end of the first pulse.

All the $x$-components are zero Eqs. (16b), (17b), (18b), and (19b) are taken from [5] but the signs have been changed due to the $-x$ pulses considered here. Figures 4 and 8 show that (16a), and (19a) are identi$\mathrm{cal}$ and are in agreement with (16b) and (19b). For $\omega_{\mathbf{Q}} \ll \omega_{\mathbf{R F}}$, our results on the echo amplitudes are consistent with the early ones.

\section{Conclusion}

With the help of Mathematica, we have extended Solomon echoes to soft pulse excitation by including the first-order quadrupolar interaction during the pulses. In addition to the four allowed echoes, the two forbidden echoes due to MQ coherences developed during the first pulse have been also predicted. Our results clearly show that the amplitudes and the shape of the echoes depend not only on the pulse lengths $t_{1}$ and $t_{3}$, but also on the quadrupolar coupling, $\omega_{\mathrm{Q}}$, and the amplitude $\omega_{\mathrm{RF}}$ of the pulse. They are valid for any ratio of $\omega_{\mathrm{Q}} / \omega_{\mathrm{RF}}$. The main restriction is that the interpulse delay $\tau_{2}$ must be short compared to the duration of FID of the central transition because its echo cannot be predicted within our hypothesis.

In order to predict the refocusing of the central transition, it is necessary to include an interaction such as the secular part of hetero- [11] or homo-nuclear [14] magnetic dipole-dipole interaction, or second-order quadrupolar shift [15], which dephase and refocus the coherences. The study of relaxation phenomena $[16-18]$ is also required. 
[1] I. Solomon, Phys. Rev. 110, 61 (1958).

[2] A. Abragam, "The Principles of Nuclear Magnetism," Oxford Univ. Press (Clarendon), Oxford 1961.

[3] M. W. Dowley, Solid State Commun. 3, 351 (1965).

[4] J. Butterworth, Proc. Phys. Soc. 86, 297 (1965).

[5] I. D. Weisman and L. H. Bennett, Phys. Rev. 181, 1341 (1969).

[6] B. C. Sanctuary and T. K. Halstead, Adv. Magn. Opt. Reson. 15, 79 (1990). G. Campolieti, B. C. Sanctuary, and H. B. R. Cole, J. Magn. Reson. 88, 457 (1990).

[7] N. Lee, B. C. Sanctuary and T. K. Halstead, J. Magn. Reson. 98, 534 (1992).

[8] "Pulsed Magnetic Resonance: NMR, ESR, and Optics," Ed. D. M. S. Bagguley, Clarendon Press, Oxford 1992.

[9] M. Mehring, E. K. Wolff, and M. E. Stoll, J. Magn. Reson. 37, 475 (1980).
[10] P. P. Man, Solid-State Nucl. Magn. Reson. 2, 165 (1993).

[11] P. P. Man, J. Chim. Phys. 89, 335 (1992); Solid-State Nucl. Magn. Reson. 1, 149 (1992); J. Magn. Reson. 100, 157 (1992); Appl. Magn. Reson. 4, 65 (1993).

[12] P. P. Man, Molec. Phys. 78, 307 (1993).

[13] P. P. Man, Molec. Phys. 72, 312 (1991).

[14] P. Mansfield, Phys. Rev. A 137, 961 (1965).

[15] D. Y. Han and H. Kessemeir, Phys. Rev. Lett. 67, 346 (1991).

[16] C. W. Chung and S. Wimperis, Molec. Phys. 76, 47 (1992).

[17] D. Petit and J. P. Korb, Phys. Rev. B37, 5761 (1988).

[18] J. Haase and E. Oldfield, J. Magn. Reson. 101 A, 30 (1993). D. Freude and J. Haase, "NMR Basic Principles and Progress,” Vol. 29, Springer-Verlag 1993. 